mesothelioma. In patients with a history of asbestos exposure, radiological imaging can be used to make a diagnosis. Accurate reporting of asbestos related lung disease is of vital importance to national statistics and affected individuals and their families may claim compensation from the government under the industrial injuries disablement benefit scheme. This study set out to examine the adequacy of reporting of asbestos related lung disease to HM coroner in a large teaching hospital.

Methods A retrospective case-note analysis was performed for all deaths occurring whilst under the care of respiratory physicians at a large teaching hospital from July 2012 to February 2013. Electronic patient records and radiological imaging for all of these patients was examined for any evidence of pleural plaques, pleural thickening, asbestos-related lung cancer, asbestosis or mesothelioma. A radiologist had reported all radiological imaging. Records were then cross-referenced with information held by the bereavement care office to identify whether these deaths had been referred to the coroner.

Results 115 patients were identified. Of these, 16 had radiological evidence of asbestos related lung disease; 4 on chest $\mathrm{x}$-ray and 12 on CT. 2 of these patients had biopsy confirmed mesothelioma. Both mesothelioma cases were referred to the coroner and a coroners' inquest subsequently performed. Only $60 \%$ of CT proven asbestos related disease and $30 \%$ of patients with CXR changes were referred. Of the cases not referred to the coroner 3 patients had bilateral pleural thickening on CXR and 4 patients had CT proven evidence of widespread pleural thickening.

Conclusions This study demonstrates that whilst reporting of malignant asbestos-related pleural disease to the coroner is adequate, there is room for improvement in the reporting of more benign disease. The authors propose that improved education of junior doctors may increase awareness of the importance of asbestos related lung disease, having far-reaching implications for both national statistics and those affected by these conditions.

\section{P208 TAKE UP OF INDUSTRIAL INJURIES DISABLEMENT BENEFITS AND SPIROMETRIC PATTERN ON SUBJECTS WITH ASBESTOS RELATED DIFFUSE PLEURAL THICKENING (DPT)}

S Gudur, J Hoyle; North Manchester General Hospital, Manchester, UK

\subsection{6/thoraxjnl-2013-204457.360}

Industrial injuries disablement benefit (IIDB) defines asbestos related diffuse pleural thickening (DPT) with obliteration of a costophrenic angle on chest $\mathrm{x}$-ray as eligible for compensation. Previous studies report significant airflow limitation and functional impairment in these patient.

Methods A database search from 08/2005 to 01/2013 for DPT as agreed by a multi-disciplinary team meeting as fulfilling the criteria for a claim. Any IIDB advice given, records of those who applied, smoking history and lung function tests at presentation were interrogated. Lung function was performed by a specialist cardio-pulmonary technician by body plethysomography. Airflow obstruction defined by ARTP/BTS criteria as FEV1/ FVC <70\% and severity of airflow obstruction by FEV1 $(50-80 \%$ predicted as mild, $30-49 \%$ predicted as moderate and $<30 \%$ as severe obstruction respectively).

Results 39/50 male subjects with DPT attended follow-up.
$100 \%$ were given written advice of their eligibility to claim IIDB.

24/39 (62\%) who were followed up applied for IIDB, with disability ranging from $15-100 \% .2$ were recorded as having less than $15 \%$ disability. Of 15 who did not apply, 4 patients' exposure was not work related and 11 chose not to apply.

4/39 (10\%) were never smokers and 30/39 (77\%) exsmokers.

Lung function results were available for $43 / 50$ at presentation (table 1).

Conclusions Our group of patients had a significantly high smoking history. Airflow limitation with DPT is the predominant lung function abnormality seen. Despite their disability, patients chose not to apply for IIDB as above.

The airflow limitation may be progressive and hence important to repeat the lung function tests over a time period to see if this deteriorates further contributing to worsening disability.

\section{REFERENCES}

1. Abnormal lung function associated with asbestos disease of the pleura, lung and both; comparitive analysis, Thorax 1991

\begin{tabular}{lll} 
Abstract P208 Table 1. Lung & Function & patterns. \\
\hline Spirometry & Total & $\%$ \\
\hline Mild obstruction $n=16$ & 20 & 46.5 \\
Moderate airflow limitation $n=4$ & & \\
Restriction of airflow & 3 & 7 \\
Mixed pattern & 3 & 7 \\
No obstruction & 17 & 39.5 \\
Total & 43 & 100 \\
\hline
\end{tabular}

\section{Investigation and management of pleural disease}

\section{P209 THE CLINICAL UTILITY OF PLEURAL LYMPHOCYTE SUBSET ANALYSIS IN UNDIAGNOSED EFFUSIONS}

${ }^{1} \mathrm{R}$ Bhatnagar, ${ }^{1} \mathrm{AO}$ Clive, ${ }^{2} \mathrm{~N}$ Zahan-Evans, ${ }^{2} \mathrm{AJ}$ Morley, ${ }^{3} \mathrm{PF}$ Virgo, ${ }^{2} \mathrm{ARL}$ Medford, ${ }^{2} \mathrm{JE}$ Harvey, ${ }^{4} \mathrm{CE}$ Hooper, ${ }^{5} \mathrm{SH}$ Otton, ${ }^{6} \mathrm{M}$ Brett, ${ }^{1} \mathrm{NA}$ Maskell; ${ }^{1}$ University of Bristol Academic Respiratory Unit, Bristol, United Kingdom; ${ }^{2}$ North Bristol Lung Centre, North Bristol NHS Trust, Bristol, United Kingdom; ${ }^{3}$ Department of Biochemistry, North Bristol NHS Trust, Bristol, United Kingdom; ${ }^{4}$ Worcestershire Acute Hospitals NHS Trust, Worcester, United Kingdom; ${ }^{5}$ Department of Haematology, North Bristol NHS Trust, Bristol, United Kingdom; ${ }^{6}$ Department of Cellular Pathology, North Bristol NHS Trust, Bristol, United Kingdom

\subsection{6/thoraxjnl-2013-204457.361}

Introduction Blood and tissue lymphocyte subsets (LS) analysis are routinely used in the diagnosis of a number of haematological conditions. Samples cost $£ 25$ to process and are technically labour intensive. The 2010 BTS pleural guidelines suggest LS may be useful in cases of suspected lymphoma, but there is no evidence supporting their utility or position in pleural diagnostic algorithms.

Methods Using a prospectively-maintained database of all undiagnosed pleural effusions, we analysed patients presenting to our service from 2009-2011. Fluid was initially sent for cytology and cell differential. Patients with $\geq 50 \%$ fluid lymphocytes at first sampling, with no definite cytological evidence of carcinoma, and who underwent a further pleural procedure, had 
a second sample sent for LS analysis. The cause of the original effusion was agreed by two independent consultants after a minimum 12 month follow-up period.

Results 395 patients with undiagnosed effusions were seen, of which $124(31 \%)$ were found to be lymphocytic on initial examination. 35(28.2\%) patients were excluded due to confirmed (non-haematological) malignancy (11 initial cytology, 24 biopsy). A further 46(37.1\%) patients were excluded with confirmed benign diagnoses including inflammatory pleuritis, heart failure and pleural infection. 39/43 (90.7\%) patients therefore had samples sent for LS analysis.

$7 / 43(16.3 \%)$ patients' effusions were diagnosed at 12 months as primarily due to lymphoma, with 5 having a previous diagnosis of such. Their characteristics are described in the table below.

LS analysis was diagnostic in 4 and negative in 35 cases. There were no false positive results. Therefore, based on these data, for determining whether there is haematological malignancy in lymphocytic pleural fluid, LS analysis has a sensitivity of $57.1 \%$, a specificity of $100 \%$, and a positive and negative predictive value of $100 \%$ and $91.4 \%$ respectively.

Conclusions LS analysis appears useful in a selected subgroup of patients presenting with undiagnosed effusions. It should only be considered in those patients with a lymphocytic effusion which shows negative initial cytology and/or no firm diagnosis established on pleural biopsy, or those with a past medical history of a lymphoma. A negative LS result does not exclude the possibility of a haematological cause for the effusion.

\begin{tabular}{|c|c|c|c|c|c|c|}
\hline Patient & Disease & Comorbidities & $\begin{array}{l}\text { History of } \\
\text { lymphoma }\end{array}$ & $\begin{array}{l}\text { LS } \\
\text { positive }\end{array}$ & $\begin{array}{l}\text { Tissue } \\
\text { obtained }\end{array}$ & $\begin{array}{l}\text { Tissue } \\
\text { diagnostic? }\end{array}$ \\
\hline 1 & DLBCL & Nil & No & No & Marrow & Yes \\
\hline 2 & DLBCL & $\mathrm{AF}$ & Yes & Yes & Marrow & No \\
\hline 3 & DLBCL & $\mathrm{CCF}, \mathrm{AF}$ & Yes & No & Node & Yes \\
\hline 4 & $\begin{array}{l}\text { Low grade } \\
\mathrm{NHL}\end{array}$ & Nil & Yes & Yes & Too frail & \\
\hline 5 & $\begin{array}{l}\text { Low grade } \\
\text { NHL }\end{array}$ & Nil & Yes & No & Too frail & \\
\hline 6 & CLL & Nil & Yes & Yes & No & \\
\hline 7 & CLL & T2DM, AF & No & Yes & Thoracoscopy & Yes \\
\hline
\end{tabular}

\section{P210 DEFINING THE MINIMAL IMPORTANT DIFFERENCE FOR THE VISUAL ANALOGUE SCALE FOR DYSPNOEA IN PATIENTS WITH MALIGNANT PLEURAL EFFUSIONS}

${ }^{1}$ EK Mishra, ${ }^{1} \mathrm{~J}$ Corcoran, ${ }^{1} \mathrm{R}$ Hallifax, ${ }^{1} \mathrm{~J}$ Stradling, ${ }^{2} \mathrm{~N}$ Maskell, ${ }^{1} \mathrm{~N}$ Rahman; ${ }^{1}$ University of Oxford, Oxford, UK; ${ }^{2}$ University of Bristol, Bristol, UK

\subsection{6/thoraxjnl-2013-204457.362}

Background Malignant pleural effusions (MPEs) cause disabling dyspnoea in over 1 million people worldwide per year. Currently recruiting and recently reported randomised controlled trials (RCTs) use the visual analogue scale for dyspnoea (VASD) to assess mean daily breathlessness in patients with MPEs (ISRCTN12852177, ISRCTN4784593, ISRCTN73255764) in order to provide evidence for the optimal method of symptom palliation. The VASD consists of a $100 \mathrm{~mm}$ line which subjects mark at a point representing their dyspnoea intensity.
Determination of the minimal important difference (MID) for the VASD in patients with MPEs is essential to interpret the results of these trials.

Methods Patients with a confirmed or suspected MPE undergoing a pleural procedure recorded their baseline VASD prior to the procedure and, 24 hours later, their post-procedure VASD and assessed their dyspnoea on a 7 point Likert scale. Age, gender, diagnosis, procedure performed and volume of fluid drained were also recorded.

Results A total of 114/123 (93\%) questionnaires were returned. Mean age of respondents was 70 years and $56 \%$ were female. Commonest malignancies were breast (41\%), mesothelioma (26\%) and non-small cell lung cancer (18\%). Procedures included: therapeutic aspiration (35\%), medical thoracoscopy (27\%), chest drain insertion (11\%), diagnostic tap (11\%) and indwelling pleural catheter insertion (8.8\%). The mean decrease in VASD in patients reporting a 'small but just worthwhile decrease' in their dyspnoea (i.e. equivalent to the MID) was $19 \mathrm{~mm}$ (95\% CI 14-24 mm). The volume of fluid required to drain to produce a change in VASD of $19 \mathrm{~mm}$ was $760 \mathrm{ml}$. Mean decrease in VASD for the different procedures were: chest drain $41 \mathrm{~mm}$; IPC insertion and drainage $41 \mathrm{~mm}$; therapeutic aspiration $31 \mathrm{~mm}$; diagnostic aspiration $19 \mathrm{~mm}$; and LAT $24 \mathrm{~mm}$.

Conclusion The MID for the VASD in patients with a MPE undergoing a pleural procedure is $19 \mathrm{~mm}$ (95\% CI $14-24 \mathrm{~mm}$ ). This value should be used when interpreting the results of RCTs in patients with MPEs using the VASD as an outcome measure and to calculate the sample size for future RCTs.

\section{P211 DOMICILIARY CARE OF PATIENTS WITH PRIMARY AND SECONDARY PNEUMOTHORACES: OUR EXPERIENCE IN AYRSHIRE, PATIENT SATISFACTION AND HEALTH ECONOMIC ANALYSIS}

${ }^{1}$ A Guhan, 'S Learmonth, ${ }^{2} \mathrm{O}$ Moseley, ${ }^{1} \mathrm{D}$ Sword, ${ }^{1} \mathrm{~F}$ Kelly; ${ }^{1}$ University Hospital Ayr, NHS Ayrshire and Arran, Ayr, United Kingdom; ${ }^{2}$ NHS Ayrshire and Arran, Ayr, United Kingdom

\subsection{6/thoraxjnl-2013-204457.363}

Background Domiciliary care (DC) of Spontaneous Pneumothoraces (SP) with an ambulatory Heimlich Valve (HV) attached to the Intercostal Chest Drain (ICD) has potential for carecloser-to-home, avoiding hospitalisation and saving healthcare resources. Wider acceptance of this practice requires demonstration of tangible benefits in terms of patient safety, patient satisfaction and cost savings.

Method In the last year (since July 2012), we established a consultant-led DC service for SP at the University Hospital Ayr, Ayrshire. All primary (PSP) or secondary (SSP) SP admitted through The Emergency Department (ED) were assessed within 48 hours for potential DC with informed consent, based on presence of Persistent Air Leak and predetermined criteria ensuring patient safety (Table:1). All DC patients had 72 hourly consultant ward reviews (CWR) with chest Xray or sooner should patients identify HV non-movement with coughing. ICD was removed when SP resolved. SP patients on DC were readmitted if concerns were identified. Patient satisfaction was assessed formally (Table 6).

Health economics: Costs were calculated from an NHS perspective by examining resource use associated with DC for SP. This figure was then compared against the potential cost-avoidance in terms of respiratory medicine bed days saved. Costs were taken from published ISD reference costs when available and local 